\title{
Synergistic Anti-arrhythmic Effects of Combined Blockade of Sodium and Ultra-rapid Delayed Rectifier Potassium Channels in Human Atria
}

\author{
Haibo Ni, Dominic G Whittaker, Wei Wang, Henggui Zhang \\ Biological Physics Group, School of Physics and Astronomy \\ The University of Manchester, Manchester, UK
}

\begin{abstract}
Atrial fibrillation $(\mathrm{AF})$ is the commonest cardiac arrhythmia. Developing effective and safe anti-AF drugs remains unmet. Atrial-selective blocking the ultra-rapid delayed rectifier potassium current $\left(I_{K u r}\right)$ and sodium channel $\left(I_{N a}\right)$ was recently reported to be potentially effective in the treatment of AF. We used computational models of human atria to assess the antiarrhythmic effects of $I_{\mathrm{Kur}}$ and $I_{\mathrm{Na}}$ blocks on human atria. A mathematical model of $I_{\text {Kur }}$ channel block was developed to describe the blocking effects of acacetin, an $I_{K u r-}$ selective blocker. $I_{N a}$ blockade was simulated using the guarded-receptor model. The effects of these channel blocks were simulated using mathematical models of human atrial myocytes and tissue. Applying acacetin rendered significant APD prolongation (by $29.3 \mathrm{~ms}$ at 1 $\mathrm{Hz}$ ). The $I_{N a}$ blocker significantly reduced peak $I_{N a}$ at fast pacing rates, but produced no effect at $1 \mathrm{~Hz}$ in both atria and ventricles. Combined actions of the two drugs further decreased peak $I_{N a}$ and prolonged APD at a fast pacing rate. These effects were also demonstrated in twodimensional simulations, where combined blockers showed reduced the life span of re-entrant excitation waves, exerting synergistic antiarrhythmic effects in the human atria, indicating that combined blocks of $I_{K u r}$ and $I_{N a}$ may be a valuable strategy for the treatment of AF.
\end{abstract}

\section{Introduction}

Atrial fibrillation (AF) is the commonest cardiac arrhythmia [1]. Developing effective and safe antiarrhythmic drugs remains challenging [1] and fraught with potential complications including promoting ventricular arrhythmias by prolonging action potential durations (APD). The atrial and ventricular fast $\mathrm{Na}+$ currents $\left(I_{\mathrm{Na}}\right)$ show different properties in the voltagedependent inactivation of the channel, mediating the possibility for atrial-selective sodium channel block [2]. The ultra-rapid delayed rectifier potassium current ( $\left.I_{\mathrm{Kur}}\right)$ carried by Kv1.5 channel contributes to repolarization in the atria but not in the ventricles [3,4]. Recent studies suggest that the atrial-selective blockade of $I_{\mathrm{Kur}}$ is a potentially effective strategy for the pharmacological treatment of AF [5]. Among the selective $I_{K u r}$ blockers, Acacetin, a natural flavone initially isolated from a traditional Chinese medicine Xuelianhua, was demonstrated to be a promising atrial-selective agent for the treatment of $\mathrm{AF}[3,5]$.

Multiple-channel blockade is receiving growing interest as empirical observations suggest that multichannel blockers generally offer more effective antiarrhythmic effects [1]. A recent study with mathematical simulations and experimental validations on (presumably healthy) canine hearts suggest that adding potassium channel block enhances the AF-selective antiAF effects obtainable from optimised $I_{\mathrm{Na}}$ blockade.

In this study we use computational models to assess the hypothesis that blocking $I_{\mathrm{Kur}}$ and $I_{\mathrm{Na}}$ might produce synergistic anti-arrhythmic effects in the chronic AF (cAF) remodelled human atria. The blockade of $I_{\mathrm{Kur}}$ and $I_{\mathrm{Na}}$ were simulated by modelling effects of Acacetin and optimised $I_{\mathrm{Na}}$ blockers using state-dependent blockade models, respectively. Single cell and tissue models were employed to simulate the anti-AF effects of individual and combined blockers.

\section{Methods}

\subsection{Modelling human atrial electrophysiology}

A recent mathematical model for human atrial electrophysiology [6] was employed to simulate the human atrial electrical activities under both sinus rhythm (SR) and cAF conditions. The formulation of $I_{\text {Kur }}$ was updated based on recently published data [4]. cAF induced electrical remodelling were incorporated based on a previously published model (AF-4 in [6]).

In simulating the effects of sodium blockers on human ventricular AP, the mathematical model published by O'Hara et al. [7] was adopted. The $I_{\mathrm{Na}}$ formulation in the ventricular model was replaced with that in [6]. 


\subsection{Modelling $I_{\mathrm{Na}}$ blocker}

Following previous a study [1] on simulating $I_{\mathrm{Na}}$ blockade, a theoretically optimized $I_{\mathrm{Na}}$ blocker was used. We employed the guarded receptor model to simulate the binding and unbinding of drug to sodium channel in a drug concentration-dependent manner. It was assumed that the drug predominantly binds to the activated and inactivated states of the sodium channel (denoted as $B_{\mathrm{A}}$ and $B_{\mathrm{I}}$, respectively). The blockade of $\mathrm{I}_{\mathrm{Na}}$ is given by:

$$
\begin{aligned}
I_{\mathrm{Na}} & =g_{\mathrm{Na}}\left(1-B_{A}-B_{I}\right) m^{3} h j\left(V-E_{N a}\right) \\
\frac{d B_{A}}{d t} & =K_{A}[D] m^{3} h j\left(1-B_{A}-B_{I}\right)-L_{A} B_{A} \\
\frac{d B_{I}}{d t} & =K_{I}[D](1-h)\left(1-B_{A}-B_{I}\right)-L_{I} B_{I}
\end{aligned}
$$

where $g_{\mathrm{Na}}$ is the channel conductance of $I_{\mathrm{Na}} ; \mathrm{B}_{\mathrm{A}}$ and $\mathrm{B}_{\mathrm{I}}$ are the fractional activated and inactivated blocks; $\mathrm{m}$ is the activation gate, $h$ and $j$ are the inactivation variables of the sodium channel; $V$ is the transmembrane potential; $E_{\mathrm{Na}}$ is the reversal potential of $\mathrm{Na}^{+} ; \mathrm{F}, \mathrm{R}$ and $\mathrm{T}$ are the Faraday's constant, universal gas constant and temperature respectively; $K_{\mathrm{A}}, K_{\mathrm{I}}$ are the binding constants and $L_{\mathrm{A}}, L_{\mathrm{I}}$ are the unbinding constants; [D] is the drug concentration; In the present study, a drug concentration of $60 \mu \mathrm{M}$ was studied.

\subsection{Modelling effects of Acacetin}

The effects of Acacetin on human atrial electrophysiology were simulated using the guarded receptor formalism. It was assumed that the drug binds to the open and closed gates of Kv1.5. Therefore, the binding and unbinding kinetics for open and closed states were functions of the binding constants, and given by:

$$
\begin{gathered}
I_{\text {Kur }}=g_{\text {Kur }, \mathrm{v}}\left(1-B_{O}-B_{C}\right) a i\left(V-E_{\mathrm{K}}\right) \\
\frac{d B_{O}}{d t}=K_{O} \exp \left(Z_{K O} \frac{V F}{R T}\right)[D] \operatorname{ai}\left(1-B_{O}-B_{c}\right) \\
-L_{O} \exp \left(-Z_{L O} \frac{V F}{R T}\right) B_{O} \\
\frac{d B_{c}}{d t}=K_{c} \exp \left(Z_{K c} \frac{V F}{R T}\right)[D](1-a) i\left(1-B_{O}-B_{c}\right) \\
-L_{c} \exp \left(-Z_{L c} \frac{V F}{R T}\right) B_{c}
\end{gathered}
$$

where $g_{\text {kur, }}$ is the conductance of the Kv1.5 channel; $B_{0}$ and $B_{C}$ are the fractional open and closed state block, respectively; $a$ and $i$ are the activation and inactivation gate variables; $V$ is the membrane voltage; $E_{\mathrm{K}}$ is the reversal potential of potassium; $F, R$ and $T$ are the Faraday's constant, universal gas constant and temperature respectively. $K_{\mathrm{O}}$ and $K_{\mathrm{C}}$ are the binding constant, and $L_{\mathrm{O}}, L_{\mathrm{C}}$ the unbinding constant. $Z_{\mathrm{KO}}, Z_{\mathrm{LO}}$, $Z_{\mathrm{KC}}, Z_{\mathrm{KC}}$ are the drug charge parameters for the corresponding binding and unbinding processes. [D] is the concentration of acacetin applied. In the present study, a concentration $3.2 \mu \mathrm{M}$ was considered.

The binding and unbinding parameters were obtained by fitting the model to the experimental data from [3], and are given in Table 1.

Table 1. Print sizes for different parts of the manuscript.

\begin{tabular}{lll}
\hline Parameter & Value & Unit \\
\hline$K_{\mathrm{O}}$ & 0.000194 & $\mu \mathrm{M}^{-1} \cdot \mathrm{ms}^{-1}$ \\
$L_{\mathrm{O}}$ & 0.000291 & $\mathrm{~ms}^{-1}$ \\
$Z_{\mathrm{KO}}$ & 0.257 & \\
$Z_{\mathrm{LO}}$ & -0.0128 & \\
$K_{\mathrm{C}}$ & 0.00249 & $\mu \mathrm{M}^{-1} \cdot \mathrm{ms}^{-1}$ \\
$L_{\mathrm{C}}$ & 0.000286 & $\mathrm{~ms}^{-1}$ \\
$Z_{\mathrm{KC}}$ & 0.327 & \\
$Z_{\mathrm{LC}}$ & 0.837 & \\
\hline
\end{tabular}

\subsection{Two-dimensional tissue models}

We performed two-dimensional simulations on a $7.5 \times 7.5 \mathrm{~cm}^{2}$ sheet of human atrial myocytes with 250 nodes along each direction. To solve the excitation wave propagation problem, the well-known mono-domain equation [6] was used, given by:

$$
\frac{\partial V}{\partial t}=\nabla \cdot(D \nabla V)-\frac{I_{i o n}}{C_{m}}
$$

where $V$ is the membrane potential, $\mathrm{D}$ is the diffusion parameter, $I_{\text {ion }}$ is the total ionic current in a single cardiomyocyte and $C_{\mathrm{m}}$ is the membrane capacitance of the cell. The tissue was modelled as homogeneous to conduction. The diffusion coefficient was chosen so that the model gives a conduction velocity of $0.7 \mathrm{~m} / \mathrm{s}$ measured using plane wave for the tissue with normal human atrial cells. The numerical method used in our previous study [6] was adopted here. The mono-domain equation was integrated at a time step of $0.02 \mathrm{~ms}$.

To initiate re-entrant waves, the cross field (S1-S2) protocol [1] was used. Life span of re-entrant excitations were quantified based on pseudo-ECG signals, which were computed using the equation as described in [8].

\section{Results}

\subsection{Effects of cAF on atrial electrophysiology}

Incorporation of cAF induced electrical remodeling to the significantly reduced APD (Figure 1A, $247 \mathrm{~ms}$ in SR versus $157 \mathrm{~ms}$ in the AF model). The calcium transient (CaT) was also markedly reduced with cAF remodeling (Figure 1B). 

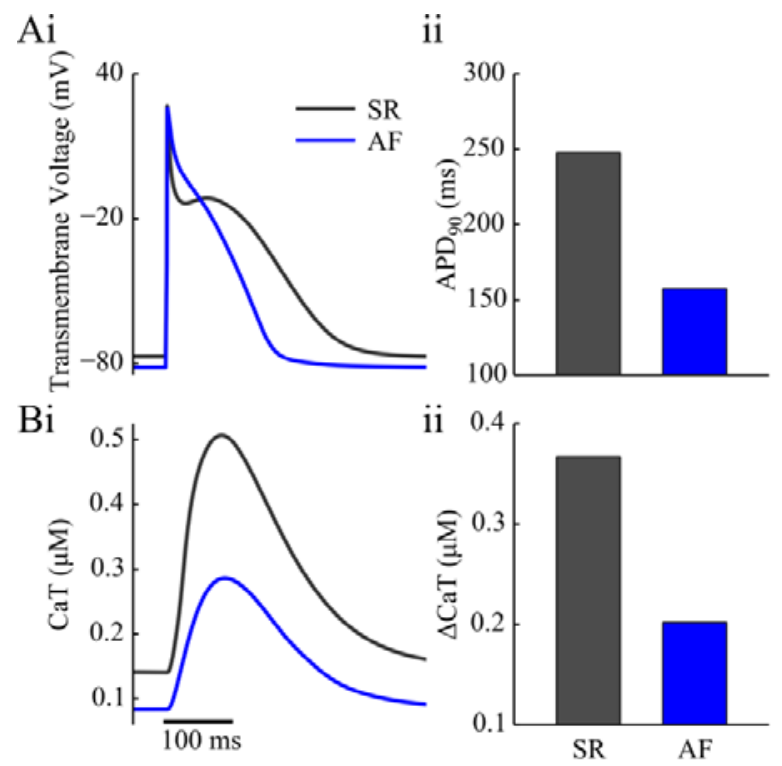

Figure 1. Simulated action potentials and calcium transient (CaT) of human atrial cardiomyocytes for sinus rhythm (SR) and cAF (AF). The action potential duration measured at $90 \%$ repolarization $\left(\mathrm{APD}_{90}\right)$ and the amplitude of $\mathrm{CaT}$ are quantified and shown in the right panels. The pacing rate was $1 \mathrm{~Hz}$.

\subsection{Simulated effects of $I_{\mathrm{Na}}$ and $I_{\mathrm{Kur}}$ blocks on the electrophysiology of human cardiomyocytes}

Individual and combined effects of $I_{\mathrm{Na}}$ and $I_{\mathrm{Kur}}$ blocks on human atrial and ventricular electrophysiology were simulated and the results are shown in Figure 2. For a slow pacing rate $(1 \mathrm{~Hz})$, blocking $I_{\mathrm{Na}}$ alone with the theoretical blocker rendered no significant effects on either AP or peak $I_{\mathrm{Na}}$ in both atrial and ventricular cells (Figure $2 \mathrm{~A} \& \mathrm{C})$. At $1 \mathrm{~Hz}$, applying acacetin prolonged APD by $29.3 \mathrm{~ms}$. Combining $I_{\mathrm{Na}}$ and $I_{\text {Kur }}$ blocks produced negligible change in APD (0.3 ms prolongation) and peak $I_{\mathrm{Na}}$.

At a fast pacing rate $(6 \mathrm{~Hz})$, alternans in APD and peak Ina were produced (Figure $2 \mathrm{Bi}$ ). Applying the $I_{\mathrm{Na}}$ blocker exerted pronounced reduction to peak $I_{\mathrm{Na}}$ and thus dVdtmax (143.7 versus $172.0 \mathrm{pA} / \mathrm{pF}$ for control, 17\% reduction); APD was prolonged by $3.5 \mathrm{~ms}$. Applying acacetin alone prolonged APD by $26.1 \mathrm{~ms}$ for big AP, and $38.1 \mathrm{~ms}$ for the small AP) and marked decrease in peak $I_{\mathrm{Na}}$ (by $28.2 \%$ for big AP, and $20.1 \%$ for small AP). More pronounced effects on AP were produced by combining $I_{\mathrm{Kur}}$ and $I_{\mathrm{Na}}$ blocks, showing more significant alternans in AP and dVdtmax; APD was prolonged by $55.8 \mathrm{~ms}$ for the big AP, demonstrating a synergistic effect in APD prolongation.
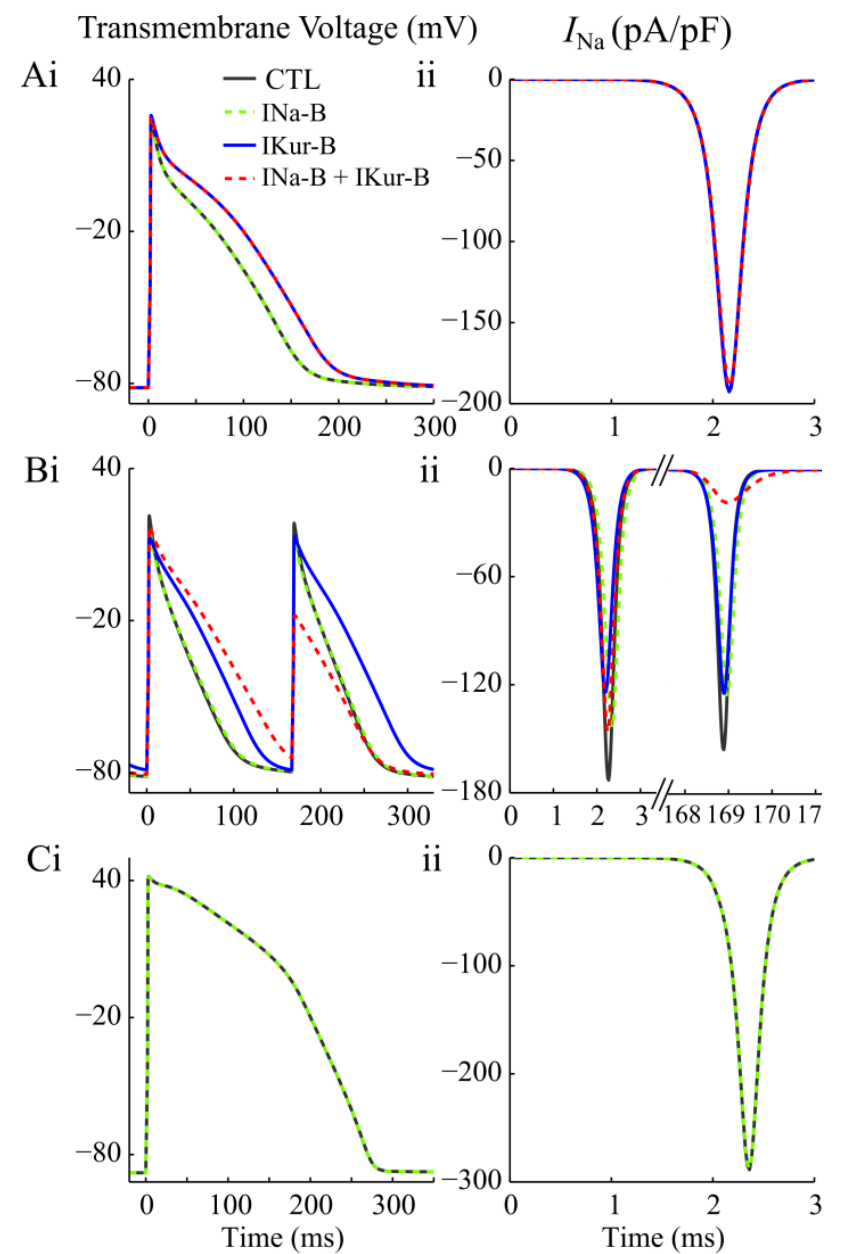

Figure 2. Simulated action potentials and $I_{\mathrm{Na}}$ for cAFremodeled atrial myocytes paced at $1 \mathrm{~Hz}(\mathrm{~A})$ and $6 \mathrm{~Hz}$ (B) for control (CTL), INa channel block (INa-B), $I_{\text {Kur }}$ channel block (IKur-B) and combined blockade of $\mathrm{I}_{\mathrm{Na}}$ and $I_{\text {Kur }}$ (INa-B + IKur-B). (C) AP and $I_{\mathrm{Na}}$ of a ventricular cardiomyocyte paced at $1 \mathrm{~Hz}$ with/without INa block. In the simulations, rate constants for INa-B were: $K_{\mathrm{A}}=100 \mathrm{~ms}^{-1} \cdot \mathrm{mol}^{-1}, K_{\mathrm{I}}=100 \mathrm{~ms}^{-1} \cdot \mathrm{mol}^{-1}, I_{\mathrm{A}}=1$ $\mathrm{ms}^{-1}, I_{\mathrm{I}}=0.01 \mathrm{~ms}^{-1}$.

\subsection{Simulated effects of $I_{\mathrm{Na}}$ and $I_{\mathrm{Kur}}$ blocks on AF termination}

Re-entrant excitation waves were initiated in the twodimensional human atrial tissue. In simulating drug blocks, drugs were applied at $2500 \mathrm{~ms}$ following initiation of reentry. Figure 3 shows the simulated pECGs, APs of a single cell in the tissue and the corresponding fractional block of $I_{\mathrm{Na}}$ and $I_{\mathrm{Kur}}$ of the same myocyte. For the control condition, reentrant waves were sustained within the simulated time of $10 \mathrm{~s}$. For INa-B, the rotors were terminated after $6552 \mathrm{~ms}$ following application of the drug. For IKur-B, the excitations diminished after $2082 \mathrm{~ms}$ following introducing acacetin. 
The application of both drugs exerted most effective termination of $\mathrm{AF}$, with the reentries were sustained for only $1052 \mathrm{~ms}$ after applying drugs.
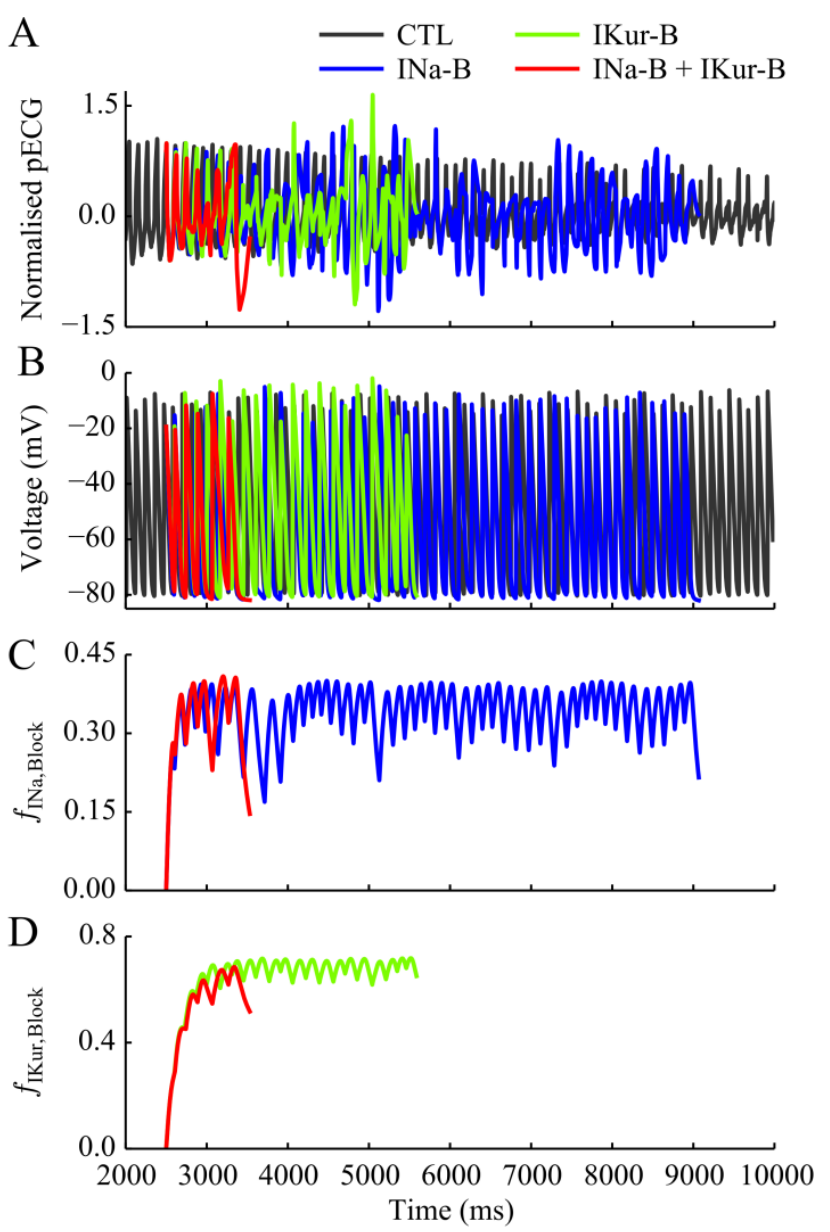

Figure 3. Simulated reentry termination by INa-B and/or IKur-B. (A) Computed pECGs; (B) AP of a single cell from the tissue during the reentrant excitations; (C) Fractional block in $I_{\mathrm{Na}}$; (D) Fraction of blocked Kv1.5 channels. For the simulation with drugs, the simulation was stopped after the termination of reentry.

\section{Discussion and conclusion}

Using biophysically detailed models of human cardiac electrophysiology, we show that state-dependent block of sodium channels exerted no effect at slow pacing rate and pronounced effects at fast rates. Combined $I_{\mathrm{Na}}$ and $I_{\mathrm{Kur}}$ blocks produced much more significant antiarrhythmic effects than an individual application of either blocker. In simulated AF, applying combined blocks was shown to be most effective in terminating rotors.

Combining $I_{\mathrm{Na}}$ blockade with $I_{\mathrm{Kur}}$ blockade produced synergistic anti-arrhythmic effects in human atria, which provides a potentially valuable strategy for the treatment of AF.

\section{Acknowledgements}

This project was supported by BHF and the University of Manchester.

\section{References}

[1] Aguilar M, Xiong F, Qi XY, Comtois P, Nattel S. Potassium Channel Blockade Enhances Atrial Fibrillation-Selective Antiarrhythmic Effects of Optimized State-Dependent Sodium Channel Blockade. Circulation 2015;132(23):2203-11

[2] Burashnikov A, Diego JMD, Zygmunt AC, Belardinelli L, Antzelevitch C. Atrium-Selective Sodium Channel Block as a Strategy for Suppression of Atrial Fibrillation Differences in Sodium Channel Inactivation Between Atria and Ventricles and the Role of Ranolazine. Circulation 2007;116:1449-57.

[3] Wu H-J, Wu W, Sun H-Y, Qin G-W, Wang H-B, Wang P, et al. Acacetin causes a frequency- and use-dependent blockade of hKv1.5 channels by binding to the S6 domain. J Mol Cell Cardiol 2011;51:966-73.

[4] Christophersen IE, Olesen MS, Liang B, Andersen MN, Larsen AP, Nielsen JB, et al. Genetic variation in KCNA5: impact on the atrial-specific potassium current IKur in patients with lone atrial fibrillation. Eur Heart J 2013;34:1517-25.

[5] Li G-R, Wang H-B, Qin G-W, Jin M-W, Tang Q, Sun H-Y, et al. Acacetin, a Natural Flavone, Selectively Inhibits Human Atrial Repolarization Potassium Currents and Prevents Atrial Fibrillation in Dogs. Circulation 2008;117:2449-57.

[6] Colman MA, Aslanidi OV, Kharche S, Boyett MR, Garratt C, Hancox JC, et al. Pro-arrhythmogenic effects of atrial fibrillation-induced electrical remodelling: insights from the three-dimensional virtual human atria. J Physiol 2013;591:4249-72.

[7] O’Hara T, Virág L, Varró A, Rudy Y. Simulation of the Undiseased Human Cardiac Ventricular Action Potential: Model Formulation and Experimental Validation. PLoS Comput Biol 2011;7:e1002061.

[8] Baher A, Qu Z, Hayatdavoudi A, Lamp ST, Yang M-J, Xie F, et al. Short-term Cardiac Memory and Mother Rotor Fibrillation. Am J Physiol - Heart Circ Physiol 2007;292(1):H180-9.

Address for correspondence.

Haibo Ni.

Room 3.08 Schuster Building, Oxford Road

The University of Manchester

Haibo.ni@manchester.ac.uk 